\title{
A case report with osteopoicileosis and collagenoma: Buschke-Ollendorff syndrome
}

Orhan Oyar ${ }^{1}$, Ali karakuzu²

Oyar O. A case report with osteopoicileosis and collagenoma: BuschkeOllendorff Syndrome. Int J Anat Var. 2019;12(3): 33-34.

Buschke-Ollendorff syndrome is an inherited disease primarily affecting the skin and bones. It is characterized by small nodular opacities osteopoikilosis in bones and collagenoma and/or elastoma in the skin. In this case report, a 22-year- old male patient with pain complaints from his martial arts was presented with the findings of physical examination and X-ray examinations. Findings related to this syndrome were discussed in this case report.

Key Words: Buschke-Ollendorff syndrome; Radiography; Hereditary disease

\section{INTRODUCTION}

B

uschke-Ollendorff syndrome is a rare hereditary disease characterized by collagenoma and/or elastoma in the skin and, osteopoikilosis derived from the words "stained bones" in Greek in bones [1]. Roberts et al. have identified Buschke-Ollendorf syndrome, indicating that osteopoikilosis may be associated with dermatofibrotic changes [2]

\section{CASE REPORT}

A 22-year-old male patient with pain and tenderness on his wrist was taken for radiography. On radiographs, uniform, multiple opacities with a millimeter diameter consistent with osteopoikilosis were noted around in the wrists, fingers and metacarps and evaluated as compatible with osteopoikilosis (Figure 1).

Considering that osteopoikilosis can be seen in various bones, PA lung radiography, pelvic radiograph, knee and ankle radiographs were obtained by including shoulder joints. In these radiographs, osteopoikilosis-compatible appearance was also observed in the vicinity of various joints such as the shoulders (Figure 2) and ankles.

During the examination of painful swellings in different parts of the body skin lesions of the right scapular region, which can be considered as connective tissue nevus in the diameters between $2-5 \mathrm{~mm}$, were detected and the collagenoma was interpreted in favor (Figure 3).

In addition, $2-5 \mathrm{~cm}$ swellings were detected in the soft tissues of the right gluteal region and left cruris in the palpation of painful areas. These nodular swellings were also evaluated by painful nodular, soft tissue ultrasonography (Figure 4).

Bilateral thoracolumbar vertebrae radiographs taken due to the present back pain were normal and there were no appearances in favor of osteopoikilosis. Thoracic magnetic resonance imaging (MRI) was performed due to the continuation of back pain and MRI showed mild hypointense on T1-weighted MR imaging (Figure 5), and hyper intense signal on T2-weighted axial (Figure 5) imaging, and showed hyper intense signal on fat suppressed T2-weighted sagital image (Figure 5) in $11^{\text {th }}$ toracal vertebra corpus. Routine blood tests and abdominal ultrasonography of the case were within normal limits.

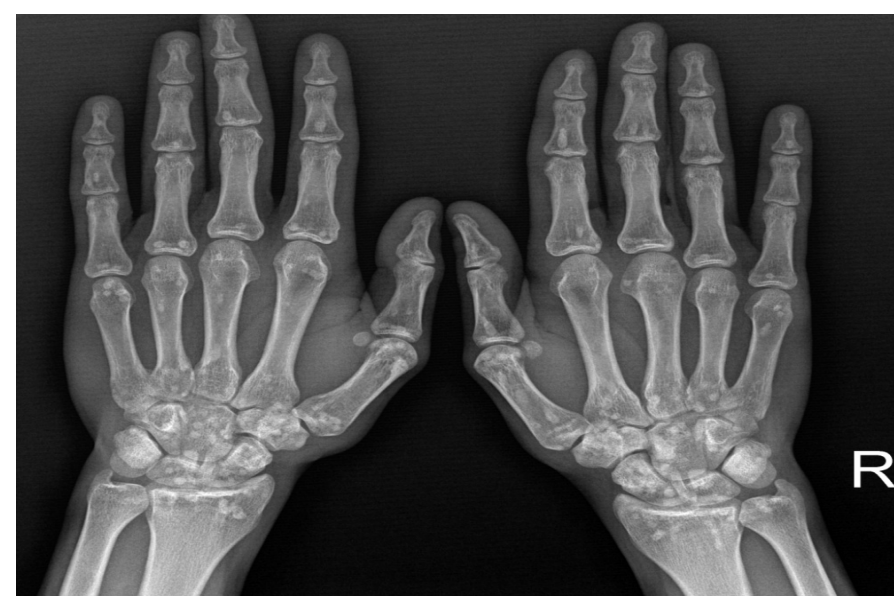

Figure 1) Hand and wrist X-ray show osteopoikilosis of the both hands and wrists.

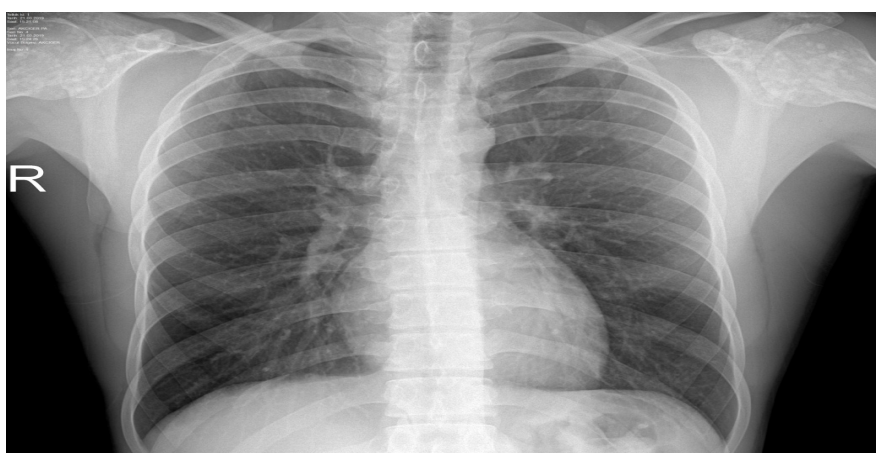

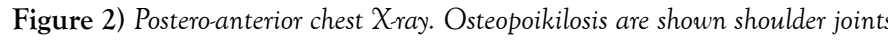
bones. Lesions do not appear in the ribs and vertebrae.

${ }^{1}$ Department of Radiology, Faculty of Medicine, Izmir Katip Çelebi University, Yesilyurt-Izmir, TURKEY; ${ }^{2}$ Department of Dermatology, Faculty of Medicine, Izmir Katip Çelebi University, Yesilyurt-Izmir, TURKEY

Correspondence: Dr. Orhan Oyar, Department of Radiology, Faculty of Medicine, Izmir Katip Çelebi University, Yesilyurt-Izmir, TURKEY Telephone+902322444444; E-mail: o_oyar@hotmail.com

Received: July 12, 2019, Accepted: Aug 14, 2019, Published: Aug 21, 2019 org/licenses/by-nc/4.0/), which permits reuse, distribution and reproduction of the article, provided that the original work is properly cited and the reuse is restricted to noncommercial purposes. For commercial reuse, contact reprints@pulsus.com 


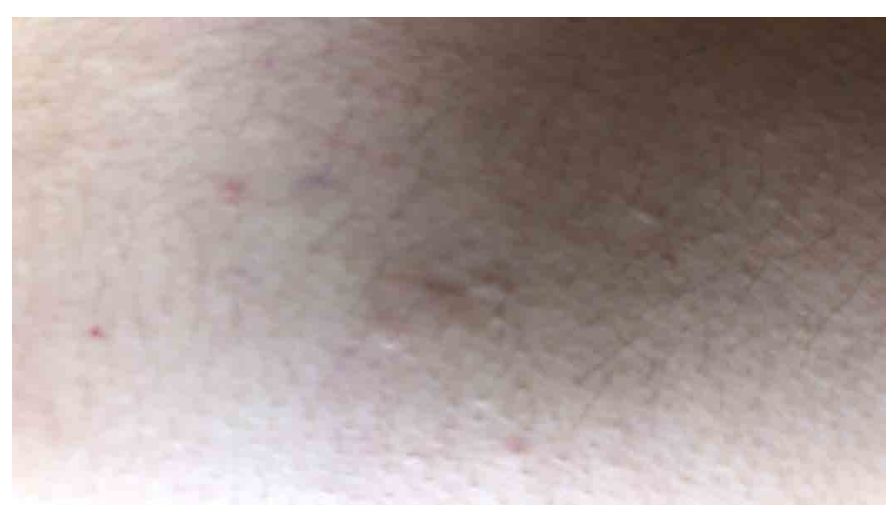

Figure 3) Skin lesions of the right scapular region.

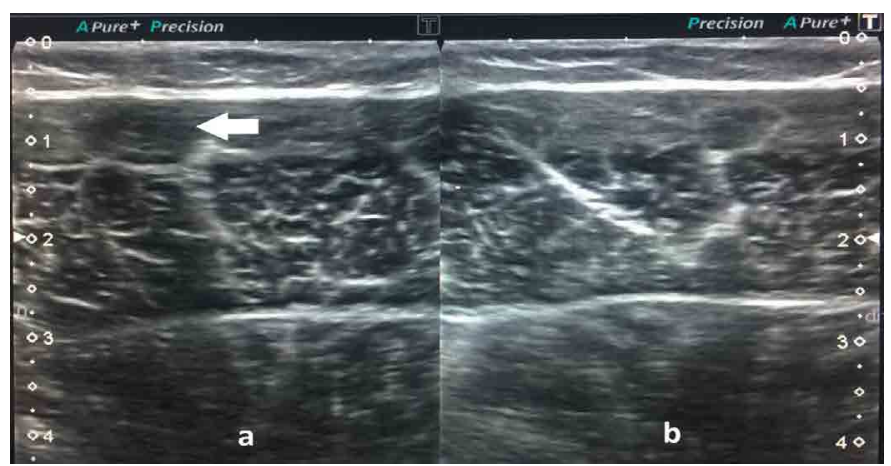

Figure 4) Comparative soft tissue ultrasonography of both cruris from posterior aspect. The left side (a) Subcutaneous tissue and muscular layer are hypertrofic compared the right side (b), and There are also hypertrophic nodular structures within the muscle layer (white arrow).
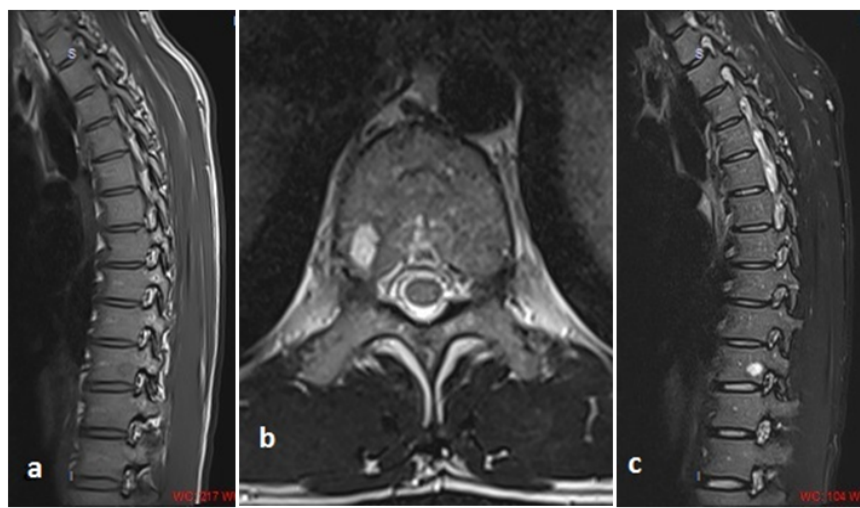

Figure 5) Vertebral hemangioma in 11th thoracal vertebrae corpus. The lesion show mild hypointensity on T1-weighted sagital MR image, high intensity on T2-weighted axial MR image, and high signal intensity continue on T2-weighted fat-suppressed sagital MR image.

\section{DISCUSSION}

Buschke-Ollendorff syndrome is a hereditary disease that affects the skin and bones, and is characterized by small spots of nodular opacity, called osteopoikilosis, derived from the words "stained bones" from the Greek, around the ends and joints of the long bones and the skin blemishes in the connective tissue. It was described by Roberts et al. [2]

The incidence of osteopoikilosis characterized by multiple tiny nodular bone islets in the bones is reported to be one in 50 thousand [3]. Osteopathia condensans disseminata also defined as osteopoikilosis is seen in all age groups; often seen in males, long bones, knees, shoulders, hips, hand-foot bones, and pelvic bones, and lesions are often symmetric and bilaterally $[2,4]$. Skull, vertebrae, costa, and mandibula involvement are seen rare. The sex of our case is male and it has symmetrical involvement in the localizations reported in the literature. There was no lesion in the vertebrae, ribs and cranium of the case.
Histopathologically, multiple compact bone islets are seen in osteopoikilosis. Therefore the cases are usually clinically asymptomatic [5]. They are detected incidentally $[4,6]$. However, the similarity of radiological appearance of such lesions should be differentiated from sclerotic-osteoblastic metastases, bone mastocytosis and tuberous sclerosis [4]. In our case, the lesions were detected incidentally with hand-wrist radiographs radiograms were taken due to trauma.

Osteopoikilois can be detected alone, but may be associated with some clinical conditions [1]. Aortic coarctation, urogenital defects and/or variations, puberty precox, some organ anomalies; endocrine disorders such as diabetes mellitus, spinal canal stenosis or dental and facial anomalies are reported as congenital accompanist anomalies with osteopoikilozis [7]. In one case, osteopoikilosis and osteosarcoma were reported to be associated with active osteogenesis [8]. In Günal-Seber-Baflaran syndrome, the association of osteopoikilosis with dacryocystitis has been demonstrated [9].

In our case, there was no problem of endocrine disorders and no organ pathologies as abdominal or thoracic region. However, there was a vertebral hemangioma in corpus of $11^{\text {th }}$ dorsal vertabra, which was not previously described in the literature. However, it is controversial whether the vertebral hemangioma is an incidental lesion or a companion of this syndrome.

Approximately $25 \%$ of the patients with osteopoikilosis have yellow-white, papular skin lesions localized on the lower back, buttocks, thighs and arms, which can show symmetry $[1,2,10]$. The most common of these lesions are the nevus in the connective tissue. The most common form of the nevi are elastomes, which are composed of a kind of flexible connective tissue. In individuals affected by this syndrome, there may be less frequently called nevus called collagenoma which develops from collagen connective tissue. Discoid lupus erythematosus and keloid may be associated lesions of this syndrome [4,11]

For these reasons, patients with osteopoikilosis should be informed about their current conditions and if possible, their radiographs and reports should be given to them. Otherwise, they may be subjected to unnecessary examination and exposure to ionizing radiation for differential diagnosis and accompanying pathologies once they apply to other centers [12].

\section{CONCLUSION}

We suggest that multiple small compact bone islets (osteopoikilosis), which we frequently encounter in daily practice, can be reported in the reports based on incidental detection of bone and skin lesions.

\section{REFERENCES}

1. https://ghr.nlm.nih.gov/condition/buschke-ollendorff-syndrome

2. Roberts NM, Langtry JA, Branfoot AC, et al. Case report: Osteopoikilosis and the Buschke-Ollendorff syndrome. Br J Radiol. 1993;66:468-70.

3. Allen DC, Grenon H. The association of Buschke-Ollendorf syndrome and nail-patella syndrome. J Am Acad Dermatol. 2002;46:621-5.

4. Resnick D, Niwayama G. Enostosis, hyperostosis and periostitis. In Resnick D (ed) Diagnosis of bone and jo-int disorders, 3rd edn. WB Saunders, Philadelphia, 1995;4404-11.

5. Szabo AD. Osteopoikilosis in a twin. Clin Orthop. 1971;79:156-63.

6. Yochum TR, Rowe LJ. In: Yochum TR, Rowe LJ, editors. Essentials of skeletal radiology 3rd edn. Lippincott Williams \& Wilkins, Baltimore, Philadelphia 2005.

7. Borman P, Ozoran K, Aydog S, et al. Osteopoikilosis: report of a clinical case and review of the literature. Joint Bone Spine. 2002;69:230-3.

8. Mindeli ER, Northup ES, Douglass HO. Osleosareoma associated with osteopoikilosis. JBJS. 1978;60:406-8.

9. Gunal I, Seber S, Basaran N, et al. Dacryocystitis associated with osteopoikilosis. Clin Genet. 1993;44:211-3.

10. Ozdemirel AE, Cakit BD, Erdem HR, et al. A rare benign disorder mimicking metastasis on radiographic examination: a case report of osteopoikilosis. Rheumatol Int. 2011;31:1113-6.

11. Bicer A, Tursen U, Ozer C, et al. Coexistence of osteopoikilosis and discoid lupus erythematosus: a case report. Clin Rheumatol. 2002;21:405-7.

12. Unluozkan F, Soyluboy FN, Taraktas A, et al. Osteopoikilozis: Olgu sunumu. Göztepe Tip Dergisi. 2014;29:146-8. 